\title{
Editorial
}

\section{Enabling Technologies towards 5G Mobile Networks}

\author{
Jing Zhao, ${ }^{1}$ Yejun $\mathrm{He}^{2}$ Jian Qiao, ${ }^{3}$ Ben Niu, ${ }^{4}$ and Qiben Yan ${ }^{5}$ \\ ${ }^{1}$ Google Inc., Mountain View, CA, USA \\ ${ }^{2}$ Shenzhen University, Shenzhen, China \\ ${ }^{3}$ Oracle Inc., Mississauga, ON, Canada \\ ${ }^{4}$ Chinese Academy of Sciences, Beijing, China \\ ${ }^{5}$ University of Nebraska, Lincoln, NE, USA \\ Correspondence should be addressed to Jing Zhao; juz139@cse.psu.edu \\ Received 9 November 2017; Accepted 9 November 2017; Published 26 November 2017 \\ Copyright (c) 2017 Jing Zhao et al. This is an open access article distributed under the Creative Commons Attribution License, \\ which permits unrestricted use, distribution, and reproduction in any medium, provided the original work is properly cited.
}

Future fifth-generation (5G) mobile networks denote the next-generation mobile networks beyond the current $4 \mathrm{G}$ mobile networks. The $5 \mathrm{G}$ networks are provisioned by the Next Generation Mobile Networks Alliance to provide much higher capacity and support various types of emerging applications with stringent quality of service (QoS) requirements. The objective of this special issue is to solicit the state-of-the-art research contributions that present key and emerging results on 5G-enabling technologies to optimize spectrum efficiency and provide heightened security and privacy.

This special issue offers a selected and articulated overview of the examined topics. It contains nine papers, and the details were listed as follows:

Shafiq et al. proposed a feature selection algorithm named "WMI_ACC," which filters most of the features with WMI metric. It further used a wrapper method to select features for ML classifiers with accuracy (ACC) metric. Their proposed algorithm can achieve 99\% flow accuracy results, which is very promising.

Mani et al. investigated channel estimation methods for multitaper GFDM (MGFDM) systems with and without discrete Fourier transform (DFT). The performance of the MMSE estimator is proved to provide a better estimate of the channel response in both cases of with and without DFT.

Zhang et al. proposed an efficient and privacy-aware power injection (EPPI) scheme suitable for advanced metering infrastructure and 5G smart grid network slice. Throughout the EPPI system, both the gateway and the utility company cannot know individual bids, and hence, user privacy is preserved.
Instead of focusing on the security architecture in the upper layer, Liu et al. investigated the secure transmission for a basic channel model in the heterogeneous network, that is, two-way relay channels. Two different approaches have been introduced and proved to be secure under three different adversarial models.

Zhao et al. described a billing scheme against free riders with several cryptographic tools, namely, the encryption scheme, signature scheme, and hash function. The proposed scheme is privacy preserving, so the charging will not disclose the locations and routine routes of each vehicle.

Liu et al. analyzed 5G development and its significant shift from a strategy policy to a user-oriented development showing the coplay of technology and society in China. They proposed a hybrid routing protocol TOHRP and a distributed channel assignment algorithm LBCA in the multichannel environment.

Cruz et al. described a peer-to-peer ( $\mathrm{P} 2 \mathrm{P})$ network that is designed to support video on demand $(\mathrm{VoD})$ services. This network is based on a video-file-sharing mechanism that classifies peers according to the window (segment of the file) that they are downloading.

Fan et al. proposed an ultralightweight mutual authentication protocol, named "ULMAP," which uses Bit and XOR operations to complete the mutual authentication and prevent the denial-of-service (DOS) attack. They also used the subkey and subindex number into the key update process to achieve the forward security. Experiment results are also involved to show its lightweight, economical, practical, and resistance to the synchronization attack. 
Fu et al. analyzed the intrusion detection requirements of IoT networks and proposed a uniform intrusion detection method for the vast heterogeneous IoT networks based on an automata model. The proposed method can detect and report the possible IoT attacks with three types: Jam-attack, False-attack, and Reply-attack automatically.

The guest editors hope the information provided in this special issue is useful and offers stimulation to the new development of 5G. Finally, we would like to thank the authors for an excellent contribution of their research works as well as we also very warmly acknowledge the reviewers for an excellent contribution of their valuable review results.

Jing Zhao

Yejun $\mathrm{He}$

Jian Qiao

Ben Niu

Qiben Yan 



\section{Hindawi}

Submit your manuscripts at

https://www.hindawi.com
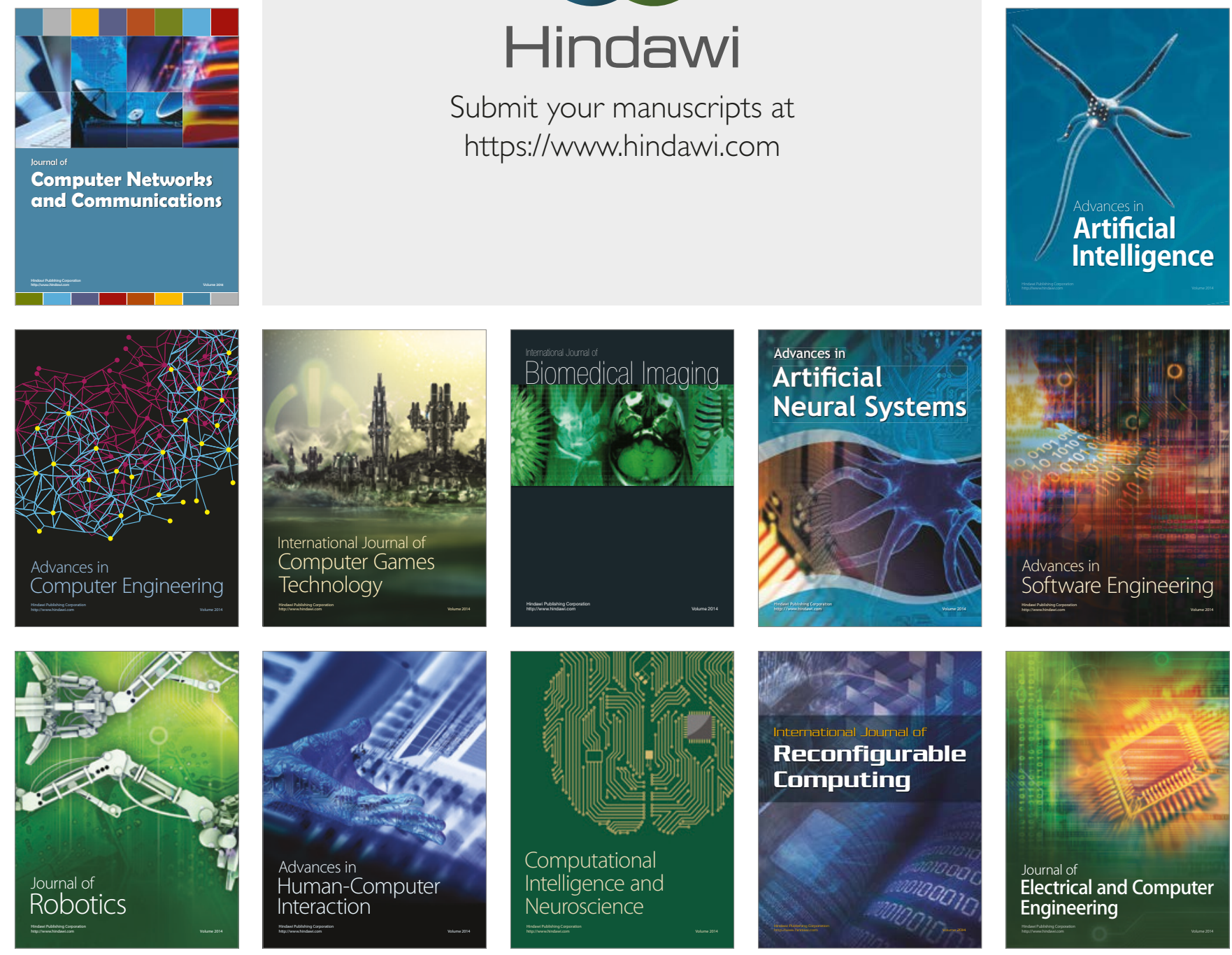